\title{
Lichen sclerosus in pediatric age: A new disease or unknown pathology? Experience of single centre and state of art in literature
}

\author{
Rossella Angotti, ${ }^{1}$ Giulia Fusi, ${ }^{2}$ Elena Coradello, ${ }^{3}$ Clelia Miracco, ${ }^{4}$ Francesco Ferrara, ${ }^{1}$ Marina Sica, ${ }^{1}$ \\ Alessandra Taddei,1 Gabriele Vasta,2 Mario Messina,1,2 Francesco Molinaro1,2 \\ 1 Pediatric Surgery, Department of Women and Children; 2 Pediatric Surgery, Department of Medical, Surgical and \\ Neurological Sciences, S. Maria alle Scotte Hospital, University of Siena, Siena, Italy; ${ }^{3}$ Department of Medical, \\ Surgical and Neurological Sciences, University of Siena; ${ }^{4}$ Pathological Anatomy Unit, Department of Medical, \\ Surgical and Neurological Science, S. Maria alle Scotte Hospital, University of Siena, Siena, Italy
}

\begin{abstract}
Lichen Sclerosus (LS) is a chronic inflammatory skin disease with unknown etiology. In pediatric age the main disease "lichenlinked" is the phimosis in male. This is a retrospective study that reports the experience of our clinic and review of the literature. We included all patients affected by pathological phimosis, treated by circumcision between January 2015 and May 2020, older than 6 years old and with an histopathological diagnosis of lichen sclerosus. The aim was to identify prognostic factors based on histological report to plan the clinical management of patients. Statistical analysis was done. We included 207 patients. The mean
\end{abstract}

Correspondence: Rossella Angotti, Pediatric Surgery, Department of Women and Children, Le Scotte Hospital, Viale Bracci 16, 53100 Siena, Italy.

Tel.: +39 0577586503; Fax +390577586174

E-mail: rossellaangotti@me.com

Key words: Lichen sclerosus; phimosis; child.

Contributions: All Authors equally contributed to collect analyzed and interpreted data. All authors read and approved the final manuscript.

Conflicts of interest: The authors certify that there is no conflict of interest with any financial organization regarding the material discussed in the manuscript.

Availability of data and materials: All data generated or analyzed during this study are included in this published article.

Ethics approval and consent to participate: Not applicable.

Informed consent: Not applicable.

Received for publication: 8 September 2021

Revision received: 25 January 2022.

Accepted for publication: 27 January 2022.

This work is licensed under a Creative Commons Attribution

NonCommercial 4.0 License (CC BY-NC 4.0).

oCopyright: the Author(s), 2022

Licensee PAGEPress, Italy

La Pediatria Medica e Chirurgica 2022; 44:275

doi:10.4081/pmc.2022.275 age of children was 9,78 years (5-18 years, $\mathrm{DS} \pm 3.29$ ). Based on the histological features we divided patients in 2 groups: early lesions $(70 / 207,34 \%)$ and advanced $(137 / 207,66 \%)$. In term of complications lichen linked we considered meatal stenosis that needed of urethral dilatations. We included 7 patients $(7 / 207$, $3,4 \%$ ). We report $P$ value Statistical Significance in many aspects. An early diagnosis of LS and surgical treatment of foreskin are essential to prevent early and late complications in children. The size of sample is a limit of the study but results encourage our management.

\section{Introduction}

Lichen Sclerosus (LS) is a chronic inflammatory skin disease with unknown etiology. LS occurs at all ages and in both sexes. The exact prevalence is unknown, however, it is estimated at 0.1 $0.7 \%$ for prepuberal pediatric age. In pediatric age the main disease "lichen-linked" is the phimosis in male. The $24 \%$ of male affected by LS is asymptomatic. The most common manifestations of LS are the presence of redness or whitish areas on the glans penis, foreskin or coronal sulcus, and thickening of the skin of the foreskin. However, they are all aspecific.

Surgical treatment (preputialplasty or circumcision) is often the main approach for LS with success rate of 76-100\%.1-7 This is a retrospective study that reports the experience of our clinic and review of the literature.

\section{Materials and Methods}

This is a retrospective study conducted at the Clinic of pediatric Surgery of Siena. The data have been extrapolated from a kept database (internal software of the hospital). All patients who underwent ritual circumcision were excluded. All patients treated by preputialplasty were excluded because in case of clinical suspicion of lichen we advise circumcision. We included all patients affected by pathological phimosis, treated by circumcision between January 2015 and May 2020, older than 6 years old and with an histopathological diagnosis of LS. We reported our experience comparing our data with literature and we considered as main aim of our study the identification of prognostic factors based on histological report to plan the clinical management of these cluster of patients. 
Statistical analysis was done by proportion test, Fisher exact test, Chi square, Pearson product moment correlation test and binomial test based on type of data.

\section{Results}

Six hundred and twelve patients underwent circumcision over 6 years. Two hundred and seven patients met criteria for inclusion in this study (207/612). We included all patients affected by pathological phimosis and underwent circumcision under general anesthesia older than 6 years old with histopathological exam of LS. The mean age of children was 9.78 years and the age range was 6 years to 18 years ( $\mathrm{DS} \pm 3.29$ ). Based on the histological features we divided patients in 2 groups: early lesions (not atrophic epidermis, subepidermic lymphocytic infiltration, ortokeratotic hyperkeratosis, absence of sclerosis) that included 70 patients $(70 / 207,34 \%)$ and advanced (atrophic epidermis above a homogenized band of dense fibrosis of the upper dermis; virtually absent inflammation) that included 137 patients $(137 / 207,66 \%$; Figure 1).

The histological exam was done by a single pathologist (CM). In term of complications lichen linked we considered meatal stenosis that needed urethral dilatation. No patient underwent dilatations during circumcision. All patients underwent a topical treatment with cortisonic cream before dilatations. We confirm the need of dilatations after uroflowmetry. We considered altered flow patterns in case of interrupted curves and visible urine stream deviation during the exam and difficulty starting stream (Liverpool Nomogram);

We included 7 patients $(7 / 207,3.4 \%)$. The mean age was 9.57 years (range 6-11 years) DS+/- 1,99. The mean number of dilatations was 1.57 (range 1-3). Two of them belonged to group A and 5 of them belonged to group B.

\section{Statistical analysis}

We used many statistical tests based on the size and type of sample and we considered many endpoints.

\section{Correlation between LS and circumcision}

The statistical test used was proportion test.

In our population the clinical suspicion of LS in patients who underwent circumcision was confirmed by histological exam (Table 1).

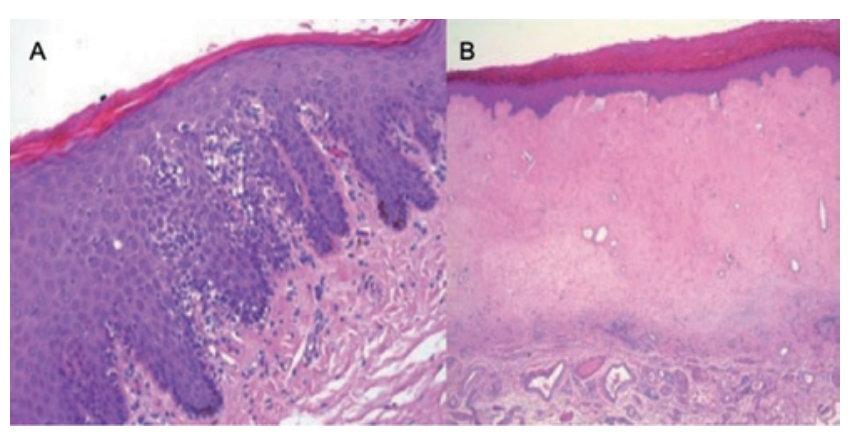

Figure 1. A) Boy, 7 years old: early lesion. Ortokeratotic hyperkeratosis; not atrophic epidermis, a sparse, subepidermal inflammatory infiltrate without fibrosis is shown; B) Boy, 8 years old: advanced lesion. Atrophic epidermis above a homogenized band of dense fibrosis of the upper dermis; virtually absent inflammation. Hematoxylin and Eosin.

\section{Correlation between presence lichen sclerosus and age}

We report that there is a correlation between LS and age (Table 2 and 3). The statistical significance decreases from 17 years even if the size of sample is lower so this can be considered a bias. We don't report a statistical significance in the sample of 18 years.

\section{Correlation between histological aspects in term of presence of Lymphocytic infiltrations and grade of lichen lesion}

Fisher exact test was used.

We confirm that the presence of lymphocytic infiltrations in prepuce is related to an early lesion with positive prognosis (Table 4).

Table 1. Correlation between LS and circumcision.

\begin{tabular}{lc}
\hline Confidence interval & $95 \%$ \\
Total case of patients circumcised & 612 \\
\hline Total case of patients circumcised & 207 \\
with lichen sclerosus (histological confirmation) & \\
Percentage of LS positives & 0.338235294 \\
\hline Max Confidence interval & 1 \\
Min Confidence interval & 0.3075518 \\
\hline P value & $2.20(\mathrm{E}-16)$ \\
P value and Statistical Significance & Yes
\end{tabular}

Table 2. Correlation between presence lichen sclerosus and age.

\begin{tabular}{lc}
\hline Confidence interval & $95 \%$ \\
Total case of patients & 207 \\
\hline Max Confidence interval & 0.78 \\
Min Confidence interval & -0.98 \\
\hline
\end{tabular}

Table 3. Correlation between presence lichen sclerosus and age: $P$ value and Statistical Significance age related.

\begin{tabular}{lcc} 
Age (years) & P value & Statistical \\
6 & $1.73(\mathrm{E}-09)$ & Significant \\
7 & $7.80(\mathrm{E}-09)$ & Significant \\
\hline 8 & $2.41(\mathrm{E}-22)$ & Significant \\
9 & $2.89(\mathrm{E}-16)$ & Significant \\
\hline 10 & $3.208(\mathrm{E}-14)$ & Significant \\
11 & $1.49(\mathrm{E}-14)$ & Significant \\
\hline 12 & $1.54(\mathrm{E}-8)$ & Significant \\
13 & 0.00015 & Significant \\
\hline 14 & $1.95(\mathrm{E}-06)$ & Significant \\
15 & $1.09(\mathrm{E}-6)$ & Significant \\
\hline 16 & 0.0019 & Significant \\
17 & 0.005 & Significant \\
\hline 18 & 0.25 & Not significant \\
\hline
\end{tabular}

Table 4. Correlation between histological aspects in term of presence of Lymphocytic infiltrations and grade of lichen lesion.

\begin{tabular}{lc}
\hline Total case of patients & $\begin{array}{r}70 \text { (group A, with presence } \\
\text { of Lymphocytic infiltrations) }\end{array}$ \\
Pvalue & 0.0005766 \\
\hline Pvalue and Statistical Significance & Yes \\
\hline
\end{tabular}




\section{Correlation between histological aspects and complications lichen linked in term of meatal stenosis and need of dilatation}

Binomial statistical test was used.

There is a known correlation in our study between lichen sclerosus advanced and meatal stenosis with needing of urethral dilatation (Table 5).

\section{Discussion}

LS is a common disease in adult age. However, to date, it must be considered also a frequent pediatric disease. 1,2 The increasing of its incidence in pediatric age could be related to the major knowledge of it as cause of pathological phimosis but also to new habits between children that favor high diffusion of infection. ${ }^{5}$ Our data respect the percentages reported in literature in term of incidence of LS in pediatric age (0.1-0.7\%) but complications lichen-linked in term of meatal stenosis is lower (3.4\% against 7-19\%). ${ }^{1-3,8}$ This last value could be associated with the small size of sample (limit of study) or with an earlier clinical diagnosis and treatment of patients. These percentages are also important epidemiological data to reveal the presence and spread of the disease in our area. According to the literature the clinic exam is the first step to identify a LS. ${ }^{1-5}$ This is well highlighted by our study, because the main indication to circumcision is the clinical suspicion of lichen that has been confirmed by histological exam in $33,8 \%$ of cases $(\mathrm{p}=9.68, \mathrm{E}-06)$.

We conclude that high level of suspicion for LS in patients with pathological phimosis older than 6 years is important. Histological exam of prepuce is imperative.

The main aim of our study was to identify prognostic factors based on histological data to stratify patients in high or low complications clusters. Indeed the planning of long-term management of patients could be a good opportunity to avoid serious complications in pediatric and adult age.

Basing on our data we report that the histological diagnosis of "early lichen" is statistically correlated with the inflammatory lymph histiocytic infiltrate $(\mathrm{p}<0.05)$ and has a better prognosis, because the incidence of complications in term of meatal stenosis in our series is low. The histological report of advanced LS is statistically significantly associated with complications $(\mathrm{p}<0.05)$ and therefore with a worse prognosis.

These data are important also in term of long-term management. Indeed based on histological exam we can plan a different follow up that to date was based only by the esthetical aspect of urethral meatus. Clinical and instrumental controls (flussometry) and repetitive dilatations under general anesthesia can have a dramatic impact to life of patients and family in term of psychological

Table 5. Correlation between histological aspects and complications lichen linked in term of meatal stenosis and need of dilatation.

\begin{tabular}{lc}
\hline Total case of patients & 7 \\
Pvalue & $9.68(\mathrm{E}-6)$ \\
\hline Pvalue and Statistical Significance & Yes \\
\hline
\end{tabular}

effects and quality of life. Furthermore, it is no negligible the economic impact for the national health system.

Based on our experience we recommend to modulate the follow up of patients related to histological exam. The patient with diagnosis of advanced LS must be followed regularly and treated by local therapy and serial urethral dilatations. For other patients, wait-and-see management is the better choice, carrying out more delayed periodic checks except in case of clinical changes.

In our clinic, to date, we follow patients for 1 years after surgery (check up at 3, 6, 12 months). However we are setting a new schedule of follow up based on histological exam. Patients with early LS should be evaluated every 6 months for 5 years; patients with advanced LS, should be valued based on the response to local therapy and serial urethral dilatations. Also we advice to all patients a long term follow up by urologist during the young adult age.

\section{Conclusions}

LS is a chronic disease with an increasing of the incidence in pediatric age. ${ }^{1-5}$ An early diagnosis and surgical treatment of foreskin are essential to prevent early complications (stenosis of the urethral meatus and urethral stenosis) and late (sexual disorders, neoplastic evolution in squamous cell carcinoma). ${ }^{8}$ This is the first study with focus on the correlation between the histological stage of the disease and long-term prognosis. We know, however, that the size of sample is small so this is a limit in term of statistical significance and we need a major data in the future. However, our results encourage us to follow this pathway.

\section{References}

1. Promm M, Rösch WH, Kirtschig G. Lichen sclerosus im Kindesalter. Urol 2020;59:271-7.

2. Tran DA, Tan X, Macri CJ, Goldstein AT et al. Lichen Sclerosus: an autoimmunopathogenic and genomic enigma with emerging genetic and immune targets. Int J Biol Sci 2019;15:1429-39.

3. Gundula K. Lichen sclerosus-presentation, diagnosis and management. Dtsch Arztebl Int 2016;113:337-43.

4. Kizer WS, Prarie T, Morey AF. Balanitis xerotica obliterans: epidemiologic distribution in an equal access health care system. South Med J 2003;96:9e11.

5. Celis S, Reed F, Murphy F et al. Balanitis xerotica obliterans in children and adolescents: A literature review and clinical series. J Pediatr Urol 2014;10:34-9.

6. Angotti R, Cerchia E, Molinaro F. et al. Circumcision with Glubran ${ }^{\circledR} 2$ in children: experience of Italian Center. Gland Surg 2016;5:391-7.

7. Angotti R, Molinaro F, Ferrara F. et al. Preputialplasty: can be considered an alternative to circumcision? When, how, why? Experience of Italian centre. Gland Surg 2018;72:228-233.

8. Homer L, Buchanan KJ, Nasr B, et al. Meatal stenosis in boys following circumcision for lichen sclerosus (balanitis xerotica obliterans). J Urol 2014;192:1784-8. 